\title{
MECHANICAL CIRCULATORY SUPPORT IN CHILDREN WITH CARDIAC DISEASE
}

Brian W. Duncan, MD

Viktor Hraska, MD

Richard A. Jonas, $\mathrm{MD}^{\mathrm{a}}$

David L. Wessel, MD ${ }^{\mathrm{b}}$

Pedro J. Del Nido, $\mathrm{MD}^{\mathrm{a}}$

Peter C. Laussen, $\mathrm{MD}^{\mathrm{b}}$

John E. Mayer, MD

Robert A. Lapierre, BS, CCP

Jay M. Wilson, MD
Objective: To review the experience from a single center that uses both extracorporeal membrane oxygenation and ventricular assist devices for children with cardiac disease requiring mechanical circulatory support. Methods: A retrospective chart review was performed for all pediatric patients with cardiac disease who required support with extracorporeal membrane oxygenation or ventricular assist devices. Statistical analysis of the impact of multiple clinical parameters on survival was performed. Results: From 1987 through 1996 we provided mechanical circulatory support for children with a primary cardiac diagnosis using extracorporeal membrane oxygenation (67 patients) and ventricular assist devices (29 patients). Twenty-seven of $67(40.3 \%)$ patients supported with extracorporeal membrane oxygenation and 12 of 29 $\mathbf{4 1 . 4 \% )}$ patients supported with ventricular assist devices survived to hospital discharge. Failure of return of ventricular function within 72 hours of the institution of support was an ominous sign in patients supported with either modality. Univariate analysis revealed the serum pH at 24 hours of support, the serum bicarbonate at 24 hours of support, the urine output over the first 24 hours of support, and the development of renal failure to have a statistically significant association with survival in children supported with extracorporeal membrane oxygenation. None of the clinical parameters evaluated by univariate analysis were significantly associated with survival in the patients supported with ventricular assist devices. Conclusions: Extracorporeal membrane oxygenation and ventricular assist devices represent complementary modalities of mechanical circulatory support that can both be used effectively in children with cardiac disease. (J Thorac Cardiovasc Surg 1999;117:529-42)
$\mathrm{M}$ echanical circulatory support after failure of conventional medical therapy is now common in patients with cardiac disease. In adult patients, left ventricular failure due to coronary artery disease has resulted in the widespread use of intra-aortic balloon counterpulsation and left ventricular assist devices (LVADs). The success of long-term support with implantable LVAD systems has led to their increasing use in the management of patients with end-stage left ventricular failure.

From the Departments of Cardiac Surgery, ${ }^{\mathrm{a}}$ Cardiology, ${ }^{\mathrm{b}}$ and Surgery, ${ }^{\mathrm{c}}$ Children's Hospital, Boston, Mass.

Received for publication Dec 29, 1997; revisions requested April 9, 1998; revisions received June 8, 1998; accepted for publication Oct 7, 1998.

Address for reprints: Jay M. Wilson, MD, Department of Surgery, Children's Hospital, 300 Longwood Ave, Boston, MA 02115.

Copyright $(\odot) 1999$ by Mosby, Inc.

$0022-5223 / 99 \$ 8.00+0 \quad \mathbf{1 2 / 1 / 9 5 0 2 1}$
Extracorporeal membrane oxygenation (ECMO) has been used less frequently for support in adult patients with cardiac disease. In pediatric patients with cardiac disease, however, ECMO has been the most commonly used form of mechanical circulatory support. A major reason for this is the ready availability of ECMO for the treatment of neonatal respiratory failure at pediatric centers. We have successfully used both ECMO and VAD systems, depending on the clinical situation, to support pediatric patients with cardiac disease. This report describes our experience with ECMO and VAD systems to provide mechanical circulatory support in children with cardiac disease.

\section{Patients and methods}

Patient population, data sources, and statistical analysis. A retrospective chart review of all patients with a primary diagnosis of cardiac disease supported with ECMO (67 patients) or a VAD (29 patients) was performed after obtaining institutional approval. The period of study was from January 


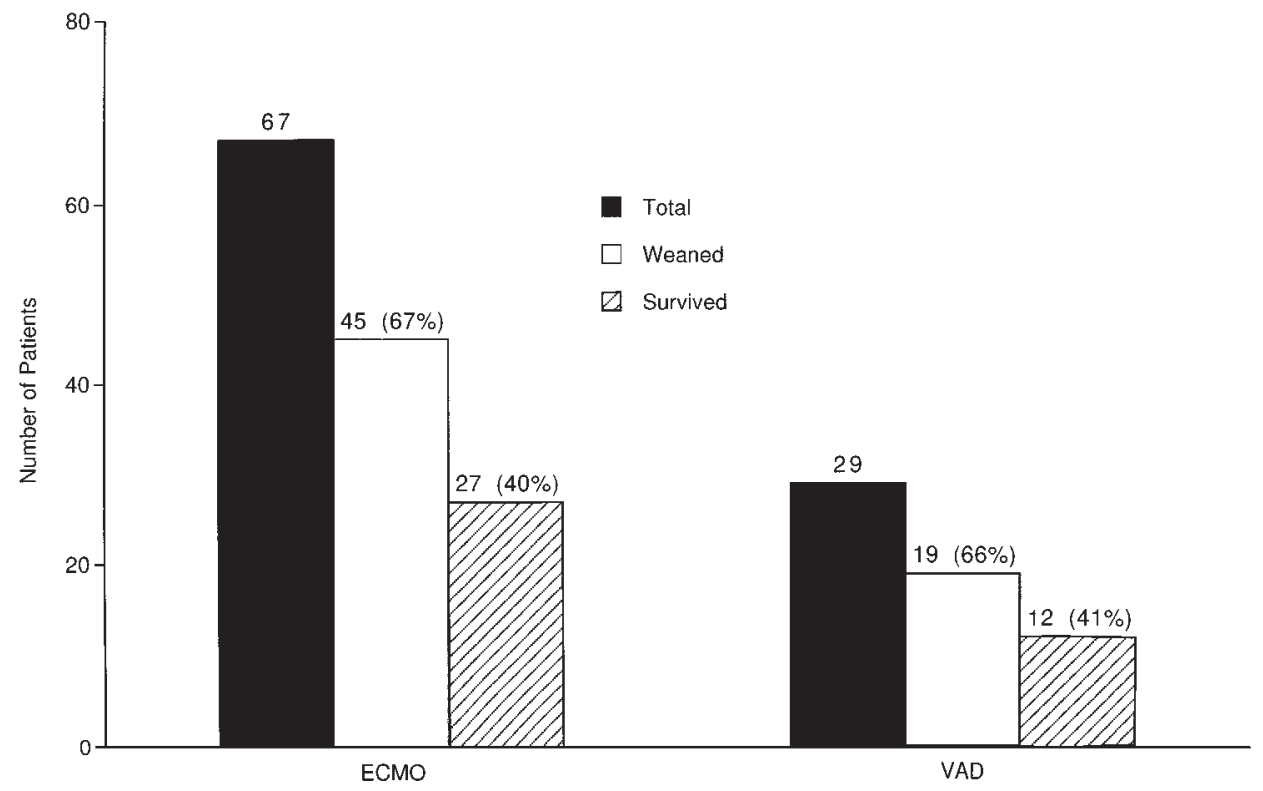

Fig 1. Total patients supported, patients weaned from support, and hospital survivors among the ECMO and VAD groups.

1987 through March 1996, comprising the entire experience at our institution using either modality for mechanical circulatory support in pediatric patients with cardiac disease.

Multiple clinical variables were analyzed for their impact on survival to hospital discharge by means of a statistical program (JMP Software, SAS Institute, Inc, Cary, NC). Two sets of variables were evaluated depending on their temporal relationship to the initiation of circulatory support with either ECMO or VAD (Appendix: Pre-support and post-support variables). These clinical variables included demographic factors, details of cardiopulmonary bypass, hemodynamics before and after support, ventilatory parameters before and after support, and details regarding the conduct of support including complications. The impact of continuous variables on the survival of patients was then assessed with parametric (Student $t$ test) or nonparametric (Wilcoxon rank sum test) tests of statistical significance. Categoric variables were assessed with Pearson's $\chi^{2}$ test. Pre-support factors with an influence on survival at a significance level of 0.10 or less were then subjected to multivariate analysis by means of a stepwise logistic regression model. Comparisons of outcomes in terms of cardiac diagnoses and indications for support for ECMO versus VAD were performed by Pearson's $\chi^{2}$ test. Comparisons of complication rates between ECMO and VAD and the influence of neck cannulation or carotid reconstruction on the incidence of neurologic complications in ECMO-supported patients were also performed with Pearson's $\chi^{2}$ test.

Components of the ECMO circuit. Techniques that we used for management of the ECMO circuit have been previously reported for neonatal and pediatric respiratory support. ${ }^{1,2}$ In brief, we used a servoregulated flow system driven by a roller pump with a membrane oxygenator (Avecor ECMO Membrane Oxygenator; Avecor Cardiovascular, Inc,
Plymouth, Minn). Pre-membrane and post-membrane in-line pressure monitors were used. A disposable heat exchanger was used to maintain constant temperature of blood in the circuit. The surface area of the oxygenator, based on the patient's size, determined priming volumes of the circuit and ranged from $350 \mathrm{~mL}$ (neonates) to $2.5 \mathrm{~L}$ (adults). Activated clotting times were maintained within 180 to 220 seconds by a continuous heparin infusion and were maintained at lower levels if significant bleeding occurred on support. Antifibrinolytic therapy with aminocaproic acid (Amicar; Lederle Parenterals, Carolina, Puerto Rico) was used in essentially all of these patients after 1990. ${ }^{1}$ Aminocaproic acid was initiated as an intravenous bolus of $100 \mathrm{mg} / \mathrm{kg}$, maintained as a continuous intravenous infusion of $30 \mathrm{mg} / \mathrm{kg}$ per hour for the initial 48 hours of support, and then discontinued.

Cannulation for ECMO. The site of cannulation for ECMO was based on the discretion of the surgeon, but in general, patients requiring support in the immediate postoperative period had direct transthoracic cannulation of the aorta and the right atrial appendage. Transthoracic cannulation was especially useful to expedite the institution of support in patients who had cardiac arrest in the postoperative period. Peripheral cannulation via the neck or femoral vessels was generally performed in patients who had not had cardiac surgery or in patients who required ECMO later in their postoperative course because of concerns about mediastinal adhesions. Neck cannulation was performed in infants and young children, whereas older children and young adults were often cannulated via the femoral vessels. Carotid reconstruction was attempted in all cases after neck cannulation; however, this was not always possible in cases in which extensive endothelial or full-thickness injury of the artery had occurred. 


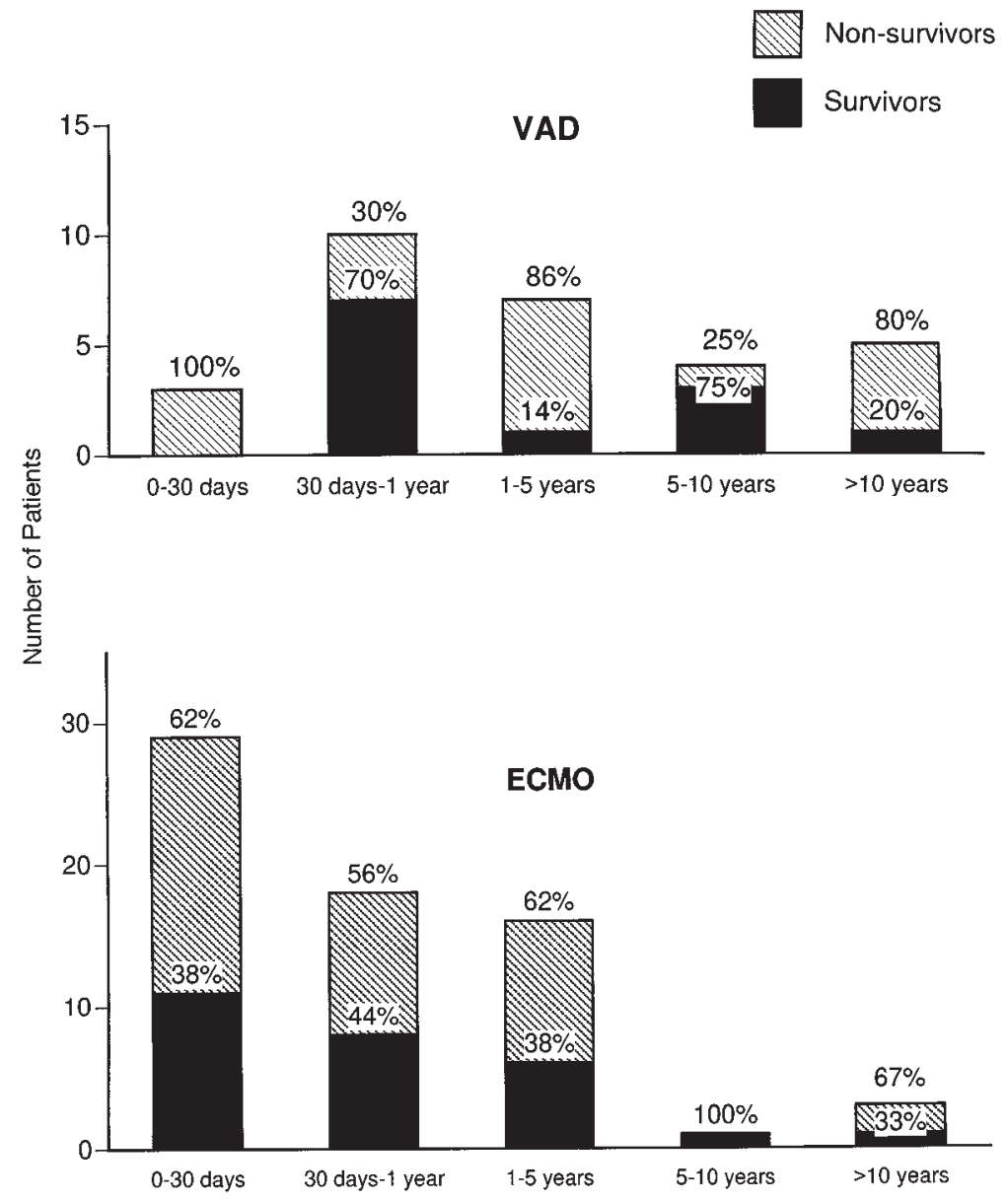

Fig 2. Age distribution for ECMO- and VAD-supported patients.

Components of the VAD circuit. Our entire experience with VADs used a centrifugal pump system (Bio-Pump; Medtronic Bio-Medicus, Minneapolis, Minn). For infants and children less than $10 \mathrm{~kg}$ the 50-mL Bio-Pump was used. For patients above $10 \mathrm{~kg}$ the $80-\mathrm{mL}$ Bio-Pump was used. The priming volume for the 50-mL Bio-Pump and $1 / 4$-inch tubing to complete the circuit was approximately $180 \mathrm{~mL}$. For the 80 $\mathrm{mL}$ Bio-Pump and $3 / 8$-inch tubing the priming volume was approximately $350 \mathrm{~mL}$. After September of 1994 we used cannulas and polyvinylchloride tubing coated with the Carmeda BioActive Surface (Medtronic Corporation, Minneapolis, Minn) in VAD circuits. The Carmeda BioActive Surface was not used for ECMO tubing and cannulas. The activated clotting time was maintained at 180 to 200 seconds, and lower activated clotting times (160-180 seconds) were maintained if tubing coated with the Carmeda BioActive Surface was used.

\section{Results}

General. From January 1987 through March 1996, 67 patients who had a primary diagnosis of cardiac disease were supported with ECMO and 29 patients with
VAD systems. These patients constituted a diverse group including cardiac surgical patients who required support in the preoperative or postoperative period, as well as cardiac medical patients who did not require other surgical intervention. Three ECMO-supported patients required 2 periods of support (runs) for a total of 70 ECMO runs. One VAD-supported patient required support with 2 runs and 1 patient required 3 runs, for a total of $32 \mathrm{VAD}$ runs.

The proportions of these patients successfully weaned from support (ECMO 45/67 [67.2\%]; VAD $19 / 29$ [65.5\%]) and those who survived to hospital discharge (ECMO 27/67 [40.3\%]; VAD 12/29 [41.4\%]) were nearly identical for the 2 modes of support (Fig 1). The median age of the ECMO-supported patients (2.6 months [range 1 day-243 months]) was considerably younger than that of the VAD-supported patients (20.2 months [range 2 days-280 months]). This was also reflected in the median weights for the 2 groups (ECMO $4.3 \mathrm{~kg}$ [range $2.4-82 \mathrm{~kg}$ ] vs VAD $9.0 \mathrm{~kg}$ [range 


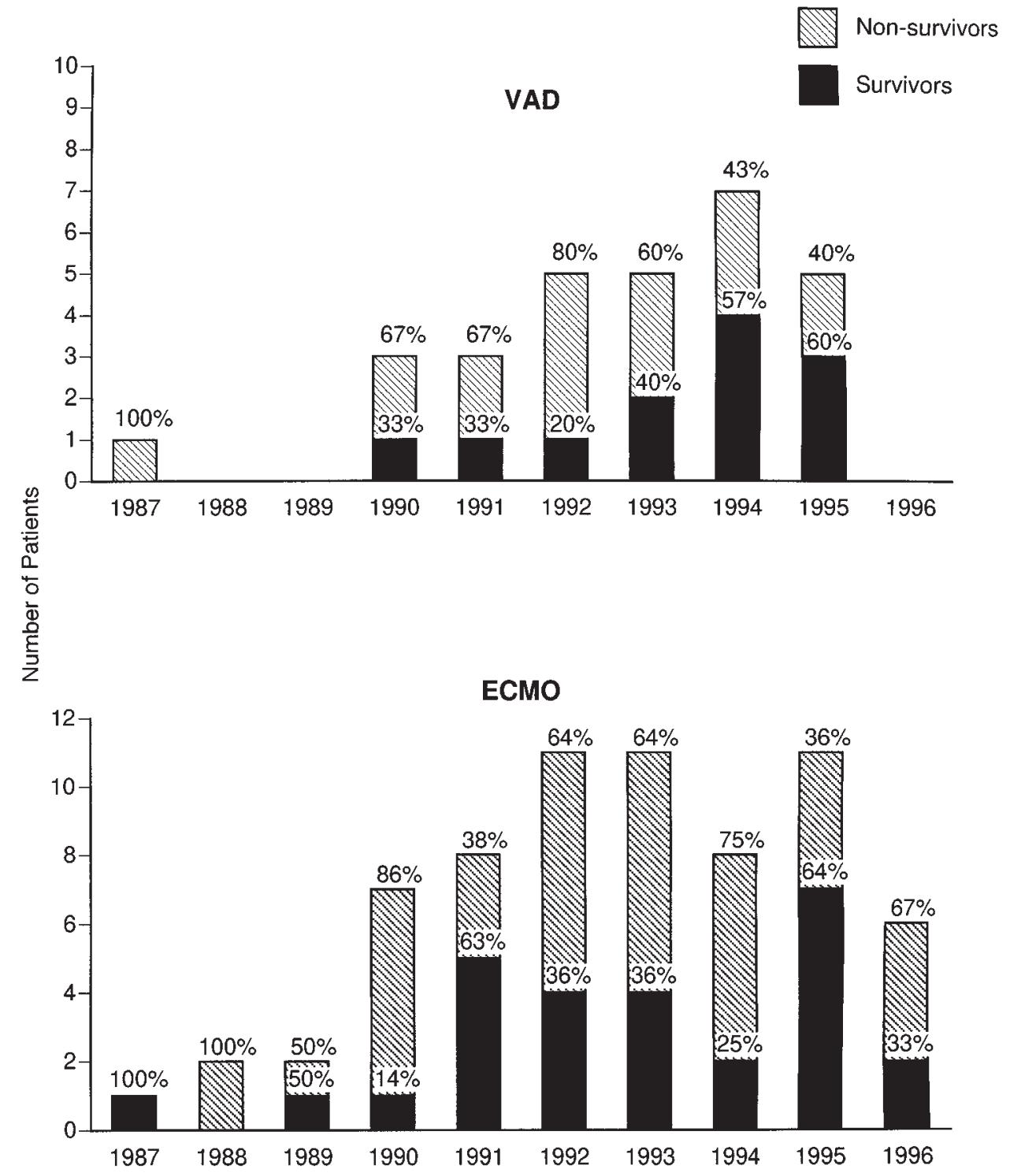

Fig 3. Annual survival for ECMO- and VAD-supported patients throughout the period of study.

2.7-71 kg]). Fig 2 demonstrates the survival by age group for ECMO and VAD. The median duration of support was longer for the ECMO-supported patients (4.8 days [range 0-29 days]) than for the VAD-supported patients (1.8 days [range 0-7.8 days]).

Over this 10-year period the number of patients undergoing mechanical circulatory support increased, with a plateau over the last 2 to 3 years (Fig 3). With 1995 as the last complete year of study, 16 children required mechanical support (11 ECMO, 5 VAD) with 10 survivors (63\%; 7 ECMO, 3 VAD). Less than 1\% of all cardiac surgical cases required mechanical circulatory support with either ECMO or VAD during the period of study.
Modes of support. Sixty-four of the 70 ECMO runs used venoarterial support, whereas 6 of the runs used venovenous support. For the 32 VAD runs there were 24 left ventricular assist device (LVAD) runs, 6 right ventricular assist device (RVAD) runs, and 2 biventricular assist device (BVAD) runs. Four patients treated with a VAD required conversion to ECMO for oxygenator support, with 1 (25\%) survivor. No patients treated by ECMO were converted to VAD after satisfactory institution of support. One patient was initially supported with intra-aortic balloon counterpulsation before the institution of ECMO and did not survive.

Cardiac diagnoses and indications for support. The survival according to cardiac diagnoses (Table I) 
Table I. ECMO and VAD: Diagnostic groups and survival

\begin{tabular}{|c|c|c|c|c|c|c|}
\hline \multirow[b]{2}{*}{ Diagnosis } & \multicolumn{3}{|c|}{ ECMO } & \multicolumn{3}{|c|}{$V A D$} \\
\hline & Survivors & Nonsurvivors & Total & Survivors & Nonsurvivors & Total \\
\hline Left-to-right shunt & $1(14.3 \%)$ & $6(85.7 \%)$ & 7 & 0 & $1(100 \%)$ & 1 \\
\hline Left-sided obstruction & $5(41.7 \%)$ & $7(58.3 \%)$ & 12 & $1(25 \%)$ & $3(75 \%)$ & 4 \\
\hline Right-sided obstruction & $1(100 \%)$ & 0 & 1 & 0 & $2(100 \%)$ & 2 \\
\hline Cyanosis, $\uparrow \mathrm{PBF}$ & $8(36.4 \%)$ & $14(63.6 \%)$ & 22 & $1(25 \%)$ & $3(75 \%)$ & 4 \\
\hline Cyanosis, $\downarrow$ PBF & $7(41.2 \%)$ & $10(58.8 \%)$ & 17 & $1(50 \%)$ & $1(50 \%)$ & 2 \\
\hline ALCAPA & $1(100 \%)$ & 0 & 1 & $5(71.4 \%)$ & $2(28.6 \%)$ & 7 \\
\hline Cardiomyopathy & $3(75 \%)$ & $1(25 \%)$ & 4 & $3(60 \%)$ & $2(40 \%)$ & 5 \\
\hline Other & $1(33.3 \%)$ & $2(66.7 \%)$ & 3 & $1(25 \%)$ & $3(75 \%)$ & 4 \\
\hline Total & $27(40.3 \%)$ & $40(59.7 \%)$ & 67 & $12(41.4 \%)$ & $17(58.6 \%)$ & 29 \\
\hline
\end{tabular}

$A L C A P A$, Anomalous left coronary artery from pulmonary artery; $P B F$, Pulmonary blood flow.

Table II. ECMO and VAD: Indications for support and survival

\begin{tabular}{|c|c|c|c|c|c|c|}
\hline \multirow[b]{2}{*}{ Indication } & \multicolumn{3}{|c|}{ ECMO } & \multicolumn{3}{|c|}{$V A D$} \\
\hline & Survivors & Nonsurvivors & Total & Survivors & Nonsurvivors & Total \\
\hline Hypoxia & $11(44 \%)$ & $14(56 \%)$ & 25 & - & - & - \\
\hline Preop. cardiac failure & $1(100 \%)$ & 0 & 1 & - & - & - \\
\hline Postop. cardiac failure & $2(28.6 \%)$ & $5(71.4 \%)$ & 7 & 0 & $2(100 \%)$ & 2 \\
\hline Cardiac arrest & $7(41.2 \%)$ & $10(58.8 \%)$ & 17 & $2(40 \%)$ & $3(60 \%)$ & 5 \\
\hline Bridge to transplantation & $2(67 \%)$ & $1(33 \%)$ & 3 & $2(50 \%)$ & $2(50 \%)$ & 4 \\
\hline Failure to wean from $\mathrm{CPB}$ & $1(10 \%)$ & $9(90 \%)$ & 10 & $7(36.8 \%)$ & $12(63.2 \%)$ & 19 \\
\hline PHT & $1(25 \%)$ & $3(75 \%)$ & 4 & - & - & - \\
\hline Failure, no operation & $2(67 \%)$ & $1(33 \%)$ & 3 & $1(50 \%)$ & $1(50 \%)$ & 2 \\
\hline Totals & $27(38.6 \%)$ & $43(61.4 \%)$ & 70 & $12(37.5 \%)$ & $20(62.5 \%)$ & 32 \\
\hline
\end{tabular}

$C P B$, Cardiopulmonary bypass; $P H T$, pulmonary hypertension.

and the indications for support (Table II) are as listed. The number of runs in Table II exceeds the number of patients in Table I because of the multiple periods of support in the 3 ECMO- and 2 VAD-supported children discussed earlier. As seen in Table II, a significant number of ECMO-supported patients required support for hypoxemia (25/70 [35.7\%]), which included a large number of children requiring support for pulmonary disease (pneumonia, pulmonary hemorrhage) irrespective of their cardiac disease. The indication for support was for cardiac arrest in 17 of 70 (24.3\%) ECMO runs and 5 of $32(15.6 \%)$ VAD runs. The survival for this critically ill subset of patients (7/17 [41\%] ECMO survivors; 2/5 [40\%] VAD survivors) was equivalent to the survival for all other indications for each modality (ECMO 20/53 [38\%], VAD 10/27 [37\%]). Three of the 7 ECMO-treated patients and 1 of the 2 VAD-treated patients surviving after pre-support cardiac arrest had cardiopulmonary resuscitation for more than 1 hour before the institution of support. Statistical analysis comparing outcomes in terms of underlying cardiac diagnosis or indications for support was performed for ECMO versus VAD. There was no difference in out- come for ECMO- or VAD-supported patients when analyzed for a given diagnostic group or specific indication for support (data not shown).

\section{Univariate analysis of survival factors}

ECMO. Table III lists those clinical parameters, from the original list that was analyzed (Appendix), found to have a $P$ value of .10 or less in a univariate analysis of survival. None of the pre-support variables attained a .05 level of statistical significance for all 67 ECMOsupported patients. Post-support variables attaining a $P$ value of .05 or less for all 67 ECMO-supported patients included the serum $\mathrm{pH}$ at 24 hours of support, serum bicarbonate at 24 hours of support, the development of severe renal failure (creatinine $>3.0 \mathrm{mg} / \mathrm{dL}$ or dialysis), and the urine output over the first 24 hours of support. The lowest $\mathrm{pH}$ at 24 hours of support for any survivor was 7.38 and the lowest serum bicarbonate at 24 hours of support for survivors was $22 \mathrm{mmol} / \mathrm{dL}$. Univariate analysis was then repeated for the same pre-support and post-support variables for the subset of 56 patients who required ECMO in the perioperative period of cardiac surgery. For this group of patients, failure to wean from cardiopulmonary bypass attained a .05 level of statisti- 
Table III. Univariate analysis of survival of patients supported by ECMO or VAD

\begin{tabular}{|c|c|c|c|}
\hline Parameter & Survivors & Nonsurvivors & $\mathrm{P}$ value \\
\hline \multicolumn{4}{|l|}{ Total ECMO } \\
\hline \multicolumn{4}{|l|}{ Pre-support variable } \\
\hline Left atrial pressure $(\mathrm{mm} \mathrm{Hg})$ & $15.0 \pm 5.5$ & $23.1 \pm 9.4$ & .058 \\
\hline \multicolumn{4}{|l|}{ Post-support variables } \\
\hline $\mathrm{pH}$ at $24 \mathrm{~h}$ & $7.51 \pm 0.07$ & $7.44 \pm 0.08$ & .003 \\
\hline $\mathrm{tCO}_{2}$ at $24 \mathrm{~h}(\mathrm{mmol} / \mathrm{dL})$ & $31.5 \pm 4.7$ & $27.1 \pm 6.8$ & .007 \\
\hline Renal failure & $2 / 27(7.4 \%)$ & $13 / 40(32.5 \%)$ & .016 \\
\hline Urine output in first $24 \mathrm{~h}(\mathrm{~mL} / \mathrm{kg} / \mathrm{h})$ & 3.2 (range 0.32-9.1) & 1.85 (range $0-9.9$ ) & .047 \\
\hline Epinephrine at $24 \mathrm{~h}(\mu \mathrm{g} / \mathrm{kg} / \mathrm{min})$ & 0 & $0.024 \pm 0.10$ & .054 \\
\hline $\mathrm{EBL}(\mathrm{mL} / \mathrm{kg})$ & 62.2 (range $0-704$ ) & 115.2 (range $0-1555$ ) & .060 \\
\hline Dopamine at $24 \mathrm{~h}(\mu \mathrm{g} / \mathrm{kg} / \mathrm{min})$ & $3.0 \pm 3.6$ & $4.9 \pm 4.8$ & .077 \\
\hline \multicolumn{4}{|l|}{ Perioperative ECMO } \\
\hline \multicolumn{4}{|l|}{ Pre-support variables } \\
\hline Failure to wean from $\mathrm{CPB}$ & $1 / 10(10 \%)$ & $9 / 10(90 \%)$ & .030 \\
\hline Left atrial pressure $(\mathrm{mm} \mathrm{Hg})$ & $15.0 \pm 5.5$ & $23.1 \pm 9.4$ & .058 \\
\hline \multicolumn{4}{|l|}{ Post-support variables } \\
\hline $\mathrm{pH}$ at $24 \mathrm{~h}$ & $7.51 \pm 0.06$ & $7.45 \pm 0.08$ & .003 \\
\hline $\mathrm{tCO}_{2}$ at $24 \mathrm{~h}(\mathrm{mmol} / \mathrm{dL})$ & $32.0 \pm 4.4$ & $27.1 \pm 6.3$ & .003 \\
\hline Dopamine at $24 \mathrm{~h}(\mu \mathrm{g} / \mathrm{kg} / \mathrm{min})$ & $2.4 \pm 2.4$ & $5.5 \pm 5.1$ & .015 \\
\hline EBL during support (mL/kg) & 98.5 (range $0-704$ ) & 161.0 (range $0-1555$ ) & .023 \\
\hline Epinephrine at $24 \mathrm{~h}(\mu \mathrm{g} / \mathrm{kg} / \mathrm{min})$ & 0 & $0.03 \pm 0.1$ & .043 \\
\hline Urine output in first $24 \mathrm{~h}(\mathrm{~mL} / \mathrm{kg} / \mathrm{h})$ & 3.1 (range 0.3-9.1) & 1.73 (range $0-9.9$ ) & .051 \\
\hline \multicolumn{4}{|l|}{ Total VAD } \\
\hline \multicolumn{4}{|l|}{ Pre-support variable } \\
\hline ALCAPA & $5 / 7(71 \%)$ & $2 / 7(29 \%)$ & .064 \\
\hline \multicolumn{4}{|l|}{ Post-support variable } \\
\hline \multicolumn{4}{|l|}{ None } \\
\hline \multicolumn{4}{|l|}{ Perioperative VAD } \\
\hline \multicolumn{4}{|l|}{ Pre-support variables } \\
\hline HCA in OR & $1 / 11(9.1 \%)$ & $10 / 11(91 \%)$ & .053 \\
\hline ALCAPA & $5 / 7(71 \%)$ & $2 / 7(29 \%)$ & .055 \\
\hline Palliative/corrective operation & $0 / 4$ & $4 / 4(100 \%)$ & .072 \\
\hline \multicolumn{4}{|l|}{ Post-support variable } \\
\hline $\mathrm{CVP}$ at $24 \mathrm{~h}(\mathrm{~mm} \mathrm{Hg})$ & $12.0 \pm 1.7$ & $19.3 \pm 4.2$ & .04 \\
\hline
\end{tabular}

$t C \mathrm{O}_{2}$, Serum bicarbonate; $E B L$, estimated blood loss; $C P B$, cardiopulmonary bypass; $A L C A P A$, anomalous left coronary artery from the pulmonary artery; $H C A$, hypothermic circulatory arrest; $O R$, operating room; $C V P$, central venous pressure.

cal significance. Significant post-support factors included the serum $\mathrm{pH}$ at 24 hours of support, serum bicarbonate at 24 hours of support, dopamine dose at 24 hours of support, estimated blood loss during support, and the epinephrine dose at 24 hours of support.

$V A D$. None of the pre-support or post-support clinical parameters tested attained statistical significance at the .05 level for all 29 VAD-supported patients. Repeating the univariate analysis for pre-support variables for the 27 patients requiring support in the perioperative period of cardiac surgery revealed the use of hypothermic circulatory arrest during the cardiac operation, the diagnosis of anomalous left coronary artery from the pulmonary artery (ALCAPA), and the nature of the operative procedure (palliative or a totally corrective operation) to have a trend toward statistical significance (Table III). Univariate analysis of the post-sup- port variables for the 27 VAD patients who were supported during the perioperative period of cardiac surgery revealed the central venous pressure at 24 hours of support to have a statistically significant impact on survival.

Multivariate analysis of survival factors. Pre-support factors that had a .10 or smaller $P$ value for all patients supported with ECMO or VAD were then analyzed with a multivariate model by means of stepwise logistic regression. Multivariate analysis was also performed on pre-support factors with a .10 level of statistical significance by univariate analysis for patients supported with either ECMO or VAD in the perioperative period. No combination of factors attained a .05 significance level with these analyses.

Transplantation experience. The patterns of use of mechanical circulatory support and outcomes for 
Table IV. Transplantation experience

\begin{tabular}{|c|c|c|c|c|}
\hline & Survivors & Diagnosis & Nonsurvivors & Diagnosis \\
\hline \multicolumn{5}{|l|}{ ECMO } \\
\hline Successful bridge & 2 & 2 (myocarditis) & 0 & \\
\hline Unsuccessful bridge & - & - & 1 & 1 (cardiomyopathy) \\
\hline Post-transplantation support & 0 & & 0 & \\
\hline Total ECMO patients & $2(66 \%)$ & & $1(33 \%)$ & \\
\hline \multicolumn{5}{|l|}{ VAD } \\
\hline Successful bridge & 2 & $\begin{array}{l}1 \text { (myocarditis), } \\
1 \text { (postcardiotomy) }\end{array}$ & 1 & 1 (myocarditis) \\
\hline Unsuccessful bridge & - & - & 1 & 1 (myocarditis) \\
\hline Post-transplantation support & 1 & 1 (myocarditis) & 1 & 1 (postcardiotomy) \\
\hline Total VAD patients & $3(50 \%)$ & & $3(50 \%)$ & \\
\hline Total patients $=9$ & $5(56 \%)$ & & $4(44 \%)$ & \\
\hline
\end{tabular}

Table V. Comparison of complication rates for ECMO and VAD runs

\begin{tabular}{lccc}
\hline Complication & ECMO incidence & VAD incidence & P value \\
\hline Hemorrhage & $28 / 70(40.0 \%)$ & $14 / 32(43.8 \%)$ & .72 \\
Central nervous system & $22 / 70(31.4 \%)$ & $4 / 32(12.5 \%)$ & .042 \\
Cardiovascular & $26 / 70(37.1 \%)$ & $13 / 32(40.6 \%)$ & .74 \\
Pulmonary & $15 / 70(21.4 \%)$ & $4 / 32(12.5 \%)$ & .28 \\
Gastrointestinal & $17 / 70(24.3 \%)$ & $1 / 32(3.1 \%)$ & .009 \\
Mechanical & $18 / 70(25.7 \%)$ & $3 / 32(9.4 \%)$ & .058 \\
Renal failure & $15 / 70(21.4 \%)$ & $3 / 32(9.4 \%)$ & .14 \\
$\quad($ dialysis or serum creatinine $\geq 3.0)$ & $19 / 70(27.1 \%)$ & $10 / 32(31.3 \%)$ & .67 \\
Any severe infection & $3 / 70(4.3 \%)$ & $2 / 32(6.3 \%)$ & .67 \\
Mediastinitis & $10 / 70(14.3 \%)$ & $1 / 32(3.1 \%)$ & .092 \\
Pneumonia & $9 / 70(12.9 \%)$ & $4 / 32(12.5 \%)$ & .96 \\
Positive blood cultures & & & \\
\hline
\end{tabular}

patients undergoing cardiac transplantation are listed in Table IV. Five patients were successfully supported with ECMO (2 patients) or VAD (3 patients) as a bridge to transplantation. Four of these patients survived to hospital discharge, whereas a single VAD-supported patient died after cardiac transplantation. In 2 patients attempted bridging to transplantation was unsuccessful (1 ECMO, 1 VAD), that is, support was initiated in anticipation of transplantation but was discontinued because of complications before a donor organ became available.

Complications. Table V compares the relative rates of complications by organ system for ECMO and VAD. For this analysis, the occurrence of a given complication was determined for each individual ECMO ( $\mathrm{n}=$ $70)$ or VAD run $(n=32)$. Gastrointestinal and neurologic complications were seen more frequently in ECMO runs than in VAD runs. Mechanical complications related to the circuit tended to be more common for ECMO than for VAD support. No complications were more frequent with VAD support.

Table VI lists the neurologic complications in these children. Intracranial hemorrhage and seizures were seen most frequently during ECMO support, whereas anoxic encephalopathy was seen in 3 of the 4 neurologic complications occurring in VAD-supported children. Table VII demonstrates that neither neck cannulation nor carotid reconstruction had any impact on the occurrence of neurologic complications with ECMO support.

Causes of death. Ventricular failure and multiple system organ failure were the most common causes of death for both ECMO- and VAD-supported patients (Table VIII).

Influence of length of time to recovery of ventricular function on survival. We examined the rate of return of ventricular function for survivors who did not undergo transplantation compared with those patients who were unable to be weaned from support (nonsurvivors and survivors who required cardiac transplantation) (Fig 4). Return of ventricular function was defined as the return of a pulsatile waveform on the peripheral arterial trace on maximal levels of support ( $80 \%$ of normal cardiac output provided by the device). Twenty-four of 25 (96\%) ECMO survivors who did not undergo transplantation had return of a pulsatile wave- 
Table VI. Type and number of neurologic complications occurring during ECMO and VAD runs

\begin{tabular}{lc}
\hline CNS complication & $\begin{array}{c}\text { No. of runs } \\
\text { (\% of total CNS complications) }\end{array}$ \\
\hline ECMO & \\
Cerebral infarction & $4(18.2 \%)$ \\
Intracranial hemorrhage & $8(36.4 \%)$ \\
Seizures & $5(22.7 \%)$ \\
Developmental delay & $1(4.6 \%)$ \\
Horner's syndrome & $1(4.6 \%)$ \\
Abnormal EEG & $1(4.6 \%)$ \\
Anoxic encephalopathy & $2(9.2 \%)$ \\
Total & 22 \\
VAD & $3(75 \%)$ \\
Anoxic encephalopathy & $1(25 \%)$ \\
Prolonged muscle weakness & 4 \\
Total & \\
\hline
\end{tabular}

$C N S$, Central nervous system; $E E G$, electroencephalogram.

Table VII. Influence of neck cannulation and carotid reconstruction on incidence of neurologic complications in ECMO-supported patients

\begin{tabular}{lcc}
\hline & CNS & \\
& complications & P value \\
\hline Neck cannulation & & .40 \\
With neck cannulation & $16 / 46(34.8 \%)$ & \\
Without neck cannulation & $6 / 24(25.0 \%)$ & \\
Carotid reconstruction & & .72 \\
With carotid reconstruction & $3 / 10(30 \%)$ & \\
Without carotid reconstruction & $13 / 26(36.1 \%)$ & \\
\hline
\end{tabular}

CNS, Central nervous system.

form at maximal levels of support by 72 hours of support. All surviving patients in the VAD group had return of ventricular function within 48 hours of the initiation of support, whereas patients in whom ejection had not returned after this point died or required cardiac transplantation.

\section{Discussion}

Several recent reports have been published concerning series in which either ECMO or VAD was used for the support of pediatric patients with cardiac disease. ${ }^{3-10}$ This report details our 10-year experience with both ECMO and VAD in children with cardiac disease as a summary of this experience and as an attempt to provide a comparison of these two modalities. These two modes of support demonstrated nearly identical rates of weaning from support and survival, suggesting that both may be used effectively and complement each other in the treatment of these children.
Table VIII. ECMO and VAD causes of death

\begin{tabular}{lrrrrr}
\hline & \multicolumn{2}{c}{$E C M O$} & & \multicolumn{2}{c}{$V A D$} \\
\cline { 2 - 3 } \cline { 5 - 6 } Cause of death & No. & $\%$ & & No. & $\%$ \\
\hline Ventricular failure & 14 & 35 & & 9 & 53 \\
Multiple system organ failure & 12 & 30 & & 4 & 23 \\
Respiratory failure & 4 & 10 & - & - \\
Anoxic brain injury & 2 & 5 & 2 & 12 \\
Intracranial hemorrhage & 2 & 5 & - & - \\
Arrhythmia & 2 & 5 & & \\
Inadequate flow rates & 2 & 5 & - & - \\
$\quad$ due to poor drainage & 1 & & & \\
Aortic cannula dislodgment & 1 & 2.5 & - & - \\
Hemorrhage (torn umbilical vein) & 1 & 2.5 & - & - \\
Hemorrhage (mediastinal) & - & - & & 6 \\
Total & 40 & $100 \%$ & & 17 & $100 \%$ \\
\hline
\end{tabular}

Cardiac diagnoses. Examining the underlying diagnoses of these patients demonstrates that the appropriate use of ECMO or VAD is based on considerations regarding the specific anatomy and physiology present in a given case. Cyanotic heart disease was present in more than half of the children who were supported with ECMO. The most common diagnoses in the VAD-supported patients were predominant univentricular failure such as ALCAPA or cardiomyopathy. We believe that ECMO is superior to VAD for the support of most children with complex congenital heart disease such as cyanotic lesions in which hypoxia, pulmonary hypertension, or biventricular failure contributes to the pathophysiology necessitating mechanical circulatory support. ECMO provides greater flexibility than VAD in these instances. In lesions in which univentricular failure predominates, such as ALCAPA, VAD provides an effective approach. ${ }^{11}$ These considerations are generally true, but we and others have successfully used VAD to support newborn infants with complex cyanotic lesions when univentricular failure predominates. ${ }^{9,10}$

Indications for support. Examination of the indications for support demonstrates the high incidence of hypoxia and pulmonary hypertension in the ECMOtreated patients. ECMO was required in several of these patients despite the use of high-frequency ventilation, nitric oxide, and liquid ventilation. These measures have been reported to decrease the need for ECMO when used to treat neonatal and pediatric respiratory failure. ${ }^{12.13}$ Our experience indicates that these adjunctive respiratory measures may fail when severe hypoxia occurs in the setting of congenital heart disease, with many of these children still ultimately requiring ECMO support.

The survival for patients who required ECMO or 


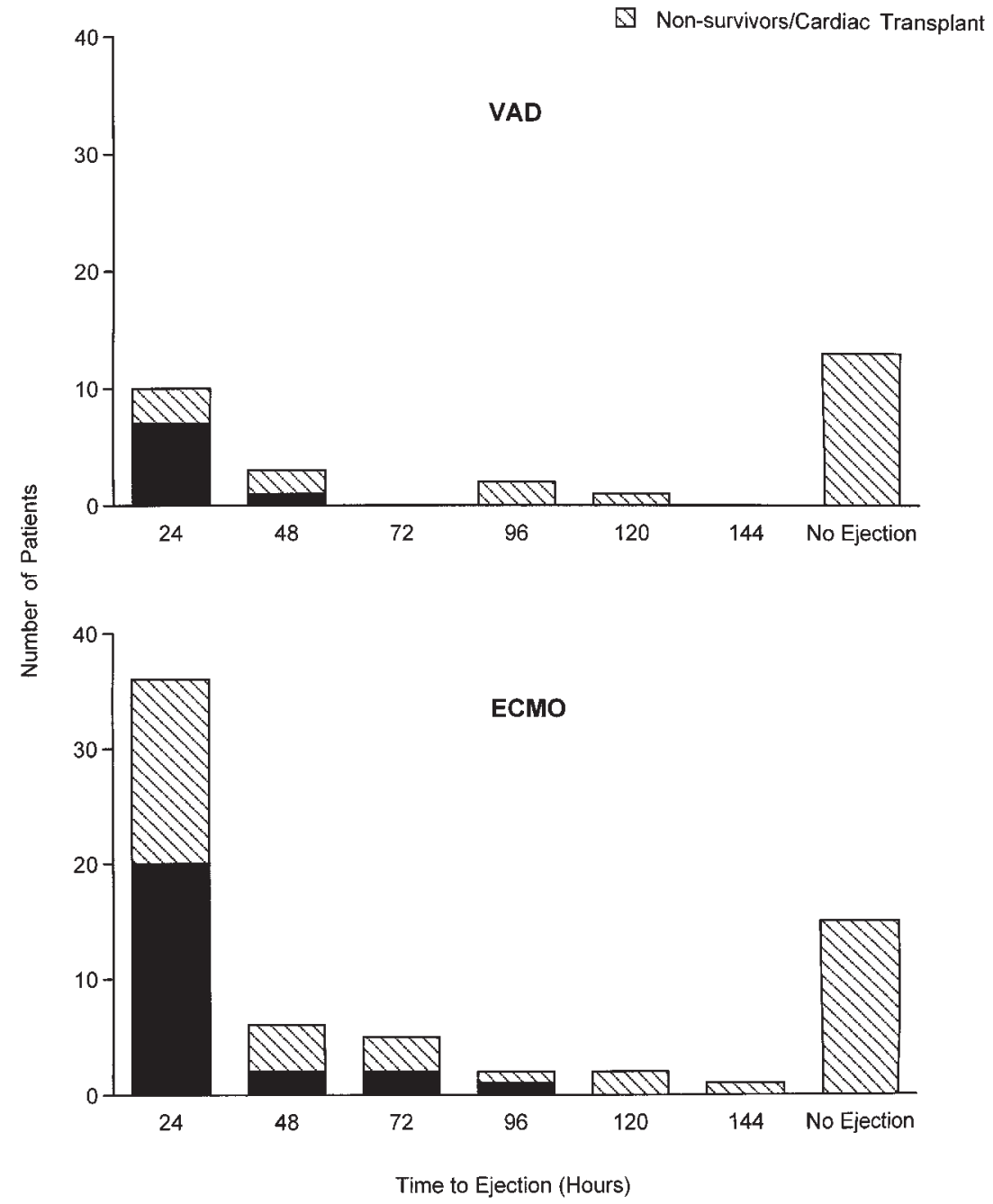

Fig 4. Time to return of ventricular function versus survival for ECMO- and VAD-supported patients.

VAD support after having a cardiac arrest was nearly identical to the survival for all other indications. These results are in agreement with earlier reports that demonstrate survival of $25 \%$ to $65 \%$ after cardiac arrest in these patients with good long-term functional status. ${ }^{14,15}$ In the surviving patients rescued with ECMO, there was no higher complication rate for any organ system. Importantly, the incidence of neurologic complications was no different from that in other survivors (data not shown).

Contraindications for support. We have not developed rigid contraindications for mechanical support but evaluate each case individually. Severe dysfunction of other organ systems (especially central nervous system) before initiation of support and extreme prematu- rity represent situations in which support would not be offered. We have successfully supported patients with a shunted single ventricle, patients with pre-support cardiac arrest, patients undergoing palliative cardiac operations, and patients with coexisting congenital diaphragmatic hernia. None of these represent absolute contraindications for support in our experience.

Determinants of survival. Determinants of the adequacy of perfusion within the first 24 hours of support as determined by the $\mathrm{pH}$, serum bicarbonate, and urine output had prognostic significance in the ECMO group. We had no survivors with a $\mathrm{pH}$ below 7.38 or a serum bicarbonate below $22 \mathrm{mmol} / \mathrm{dL}$ at 24 hours of support. Inasmuch as cardiac output was presumably normalized by the institution of support, abnormal values for 
these variables measured at this early point probably reflected the impact of significant hypoperfusion in the period before the institution of ECMO, rather than ongoing systemic hypoperfusion. The requirement of significant pressor support, with dopamine or epinephrine, to maintain perfusion during perioperative ECMO carried a poor prognosis.

Patients requiring ECMO support for failure to wean from cardiopulmonary bypass had a $90 \%$ mortality rate. Although not a statistically significant improvement in outcome, VAD-supported patients who could not be weaned from cardiopulmonary bypass fared somewhat better, with 7 survivors of 19 total patients $(37 \%)$. A major difference in these 2 groups was due to the influence of the LVAD-supported patients with ALCAPA, many of whom cannot be weaned from cardiopulmonary bypass, yet have an overall good outlook with mechanical circulatory support. ${ }^{11}$ The predominance of younger patients with complex cyanotic lesions was largely responsible for the poorer outcome of patients with ECMO support instituted in the operating room. Failure to wean from cardiopulmonary bypass is not a contraindication for ECMO support, but its use in younger patients with complex anatomy should be selective in this setting because of an appreciation of the poor outlook for this group.

Conclusions were more difficult to draw in the VADsupported group because of their smaller numbers; however, right-sided filling pressures on support, the use of circulatory arrest during the cardiac operation, and the curative or palliative nature of the cardiac repair were important clinical parameters for outcome in patients supported in the perioperative period. Right ventricular failure manifest by an elevated central venous pressure in LVAD-supported patients should suggest the need for ECMO or BVAD. The need for hypothermic circulatory arrest for operative repair implies a complex procedure on a newborn patient. As previously discussed, newborn patients with complex heart disease are often best supported with ECMO.

Complications. Although hemorrhagic complications were the most common complication in both groups, excessive blood loss was a statistically significant risk factor for death only in the ECMO-supported patients. Risk factors for excessive bleeding in our patients included chest cannulation and the need for support in the operating room (data not shown). We have previously described the use of aminocaproic acid to control bleeding while on ECMO, ${ }^{1}$ which we also use for VAD-supported patients. We have used aprotinin and tranexamic acid only sporadically.

Considered by organ system, only renal failure in
ECMO-supported patients had a significant negative impact on survival. Neurologic complications were more common during ECMO support than during VAD support. Neurologic complications were not associated with higher mortality in these patients but obviously represent a source of great morbidity. Much of the increased incidence of neurologic complications in the ECMO-supported patients can be attributed to higher rates of intracranial hemorrhage. This higher incidence of intracranial bleeding reflects the younger age of the ECMO-supported patients, including a large number of newborn infants in addition to the higher levels of anticoagulation required for the ECMO circuit. The higher incidence of central nervous system complications was independent of carotid cannulation or reconstruction.

The relatively higher rate of mechanical complications in the ECMO group is due to the increased complexity of the circuit. The presence of the oxygenator itself is a significant source of morbidity resulting in trauma to blood elements and activation of systemic inflammatory and coagulation cascades. In addition, multiple connector sites required in the ECMO circuit increase the risk of air and particulate embolism, and oxygenator failure requires interruption of flow and replacement. Complications related to the circuit were not associated with a significantly higher mortality.

Survival and length of time until return of ventricular function. We observed that ventricular function returned early after the institution of support in survivors who did not require transplantation. Lack of return of ventricular function within 48 to 72 hours was an ominous sign in our experience. We have used these results as additional prognostic data for these children. Patients without return of ventricular function within 48 to 72 hours of support are currently considered for transplantation or termination of support if there are contraindications to transplantation. Delaying this decision while awaiting return of ventricular function beyond the first 48 to 72 hours of support is not justified according to our data. Because of the scarcity of organ donors in the pediatric population, early consideration for transplantation optimizes the chances of successful organ procurement. Over this 10-year period only seven patients were supported with ECMO or VAD in an attempt to bridge them to transplantation, with five patients having successful transplantation. Our current practice of listing these children for transplantation when ventricular function has not returned after 48 to 72 hours may increase the number of patients who are successfully bridged to transplantation.

Cardiac failure and multiple system organ failure were the major causes of death in both ECMO- and VAD-sup- 
ported patients. For both of these conditions, early and aggressive consideration for transplantation may lead to higher salvage rates. We have maintained patients for as long as 4 weeks with mechanical circulatory support; however, the development of infectious complications and multiple system organ failure eventually supervenes. The use of pulsatile circulatory perfusion devices for long-term support in children may delay the development of this process. Although no pulsatile flow systems are currently available in the United States for chronic pediatric circulatory support, the development of such devices will be a welcome addition to the therapeutic options for these critically ill children.

Selection of the appropriate modality of support: ECMO versus VAD. Table IX is a "scorecard" reflecting the relative advantages and disadvantages of ECMO and VAD in the treatment of pediatric patients with cardiac disease. These general points may aid in selecting the appropriate mode of support in a given clinical setting. Pediatric centers have extensive experience with ECMO for the treatment of neonatal respiratory failure where expansion of its use to include patients with cardiac disease is often easily accomplished. Because of the absence of an oxygenator, VAD circuits are simpler, require less anticoagulation, and result in less blood trauma. ECMO may be instituted peripherally with neck or groin cannulation, whereas VAD requires a sternotomy. Left ventricular decompression is effectively performed by LVAD or BVAD; in patients supported with ECMO, the occurrence of left ventricular distention requires aggressive monitoring and prompt treatment with left atrial venting or balloon atrial septostomy. ALCAPA is the paradigm lesion successfully supported with LVAD, whereas ECMO provides greater flexibility in dealing with some forms of complex congenital heart disease in which pulmonary hypertension and hypoxia contribute significantly to the pathophysiology. Finally, biventricular support is easier to institute with ECMO, which requires only 2 cannulation sites compared with 4 cannulation sites required for BVAD. Because of size constraints, this is an important consideration when biventricular support is required in neonates.

\section{Summary}

ECMO and VAD represent complementary modalities to provide mechanical circulatory support in children with cardiac disease in whom conventional medical forms of management have failed. The two modalities are equally efficacious when used for the appropriate diagnosis and indication. ECMO was most often useful for complex congenital cardiac lesions, whereas ALCA-
Table IX. Score card for appropriate modality of support: ECMO versus VAD

\begin{tabular}{lcc}
\hline & $E C M O$ & $V A D$ \\
\hline $\begin{array}{l}\text { Experience in pediatric centers } \\
\text { Simplicity of circuit }\end{array}$ & $\checkmark$ & $\checkmark$ \\
Peripheral cannulation & $\checkmark$ & $\checkmark$ \\
Left ventricular decompression & & $\checkmark$ \\
$\begin{array}{l}\text { ALCAPA } \\
\text { Treatment of pulmonary hypertension } \\
\text { and hypoxia }\end{array}$ & $\checkmark$ & \\
Biventricular support in neonates & $\checkmark$ &
\end{tabular}

ALCAPA, Anomalous left coronary artery from the pulmonary artery.

PA represents the paradigm lesion for LVAD support in children. Early indicators of the adequacy of perfusion during support, blood loss during support, and the development of renal failure had a significant impact on survival in ECMO-supported patients. Hemorrhage was the most frequent complication for both modalities of support. Neurologic complications were seen more frequently in ECMO- as opposed to VAD-supported patients. We advocate early consideration for transplantation in patients in whom ventricular function does not recover within 48 to 72 hours after the institution of support. Because of the scarcity of organ donors in the pediatric population, early listing for transplantation optimizes the chances of successful organ procurement. Earlier consideration for transplantation should have a significant impact on the most common causes of death in these patients, namely, cardiac failure and multiple system organ failure.

We thank Kimberlee Gauvreau, ScD, for her invaluable assistance in the statistical analysis of these data.

\section{REFERENCES}

1. Wilson JM, Bower LK, Fackler JC, Beals DA, Bergus BO, Kew SV. Aminocaproic acid decreases the incidence of intracranial hemorrhage and other hemorrhagic complications of ECMO. J Pediatr Surg 1993;28:536-41.

2. Bartlett RG, Andrews AF, Toomasian JM, Haiduc NJ, Gazzaniga $\mathrm{AB}$. Extracorporeal membrane oxygenation for newborn respiratory failure. Surgery 1982;92:425-33.

3. Black MD, Coles JG, Williams WG, Rebeyka IM, Trusler GA, Bohn D, et al. Determinants of success in pediatric cardiac patients undergoing extracorporeal membrane oxygenation. Ann Thorac Surg 1995;60:133-8.

4. Walters HL, Hakimi M, Rice MD, Lyons JM, Whittlesey GC, Klein MD. Pediatric cardiac surgical ECMO: multivariate analysis of risk factors for hospital death. Ann Thorac Surg 1995; 60:329-37.

5. Dalton HJ, Seiwers RD, Fuhrman BP, del Nido PJ, Thompson $\mathrm{AE}$, Shaver MG, et al. Extracorporeal membrane oxygenation for 
cardiac rescue in children with severe myocardial dysfunction. Crit Card Med 1993;21:1020-8.

6. Meliones JN, Custer JR, Snedecor S, Moler FW, O'Rourke P, Delius RE. Extracorporeal life support for cardiac assist in pediatric patients. Circulation 1991;84(Suppl):III168-72.

7. Raithel SC, Pennington DG, Boegner E, Fiore A, Weber TR. Extracorporeal membrane oxygenation in children after cardiac surgery. Circulation 1992;86(Suppl):II305-10.

8. Weinhaus L, Canter C, Noetzel M, McAlister W, Spray TL. Extracorporeal membrane oxygenation for circulatory support after repair of congenital heart defects. Ann Thorac Surg 1989; 48:206-12.

9. Karl TR, Sano S, Horton S, Mee RBB. Centrifugal pump left heart assist in pediatric cardiac operations. J Thorac Cardiovasc Surg 1991;102:624-30.

10. Karl TR. Extracorporeal circulatory support in infants and children. Semin Thorac Cardiovasc Surg 1994;6:154-60.

11. del Nido PJ, Duncan BW, Mayer JE Jr, Wessel DL, LaPierre RL, Jonas RA. Left ventricular assist device significantly improves survival in children with anomalous left coronary artery and left ventricular dysfunction. Ann Thorac Surg. In press.

12. The Neonatal Inhaled Nitric Oxide Study Group. Inhaled nitric oxide in full-term and nearly full-term infants with hypoxic respiratory failure. N Engl J Med 1997;336:597-604.

13. Roberts JD, Fineman JR, Morin FC, Shaul PW, Rimar S, Schreiber MD, et al. Inhaled nitric oxide and persistent pulmonary hypertension of the newborn. N Engl J Med 1997;336: 605-10.

14. del Nido PJ, Armitage JM, Fricker FJ, Shaver M, Cipriani L, Dayal G, et al. Extracorporeal membrane oxygenation support as a bridge to pediatric heart transplantation. Circulation 1994;90(Pt 2):II66-9.

15. Dembitsky WP, Moreno-Cabral RJ, Adamson RM, Daily PO. Emergency resuscitation using portable extracorporeal membrane oxygenation. Ann Thorac Surg 1993;55:304-9.

\author{
Appendix: Clinical parameters evaluated for \\ impact on survival \\ Pre-support variables \\ Demographics: \\ Sex \\ Weight \\ Age \\ Intraoperative features: \\ Cardiopulmonary bypass time \\ Aortic crossclamp time \\ Circulatory arrest time \\ Hemodynamics before initiation of support: \\ Central venous pressure \\ Right atrial pressure \\ Left atrial pressure \\ Systolic blood pressure \\ Diastolic blood pressure \\ Mean arterial blood pressure \\ Arterial blood gases before initiation of support: \\ Oxygen tension \\ Carbon dioxide tension \\ $\mathrm{pH}$
}

Serum bicarbonate

Mixed venous oxygen saturation

Ventilator settings before initiation of support:

Inspired oxygen fraction

Peak inspiratory pressure

Positive end-expiratory pressure

Tidal volume

Ventilatory rate

Inotropic doses before initiation of support:

Dopamine

Amrinone

Dobutamine

Epinephrine

Site of cannulation:

Neck

Chest

Groin

Miscellaneous:

Urine output before support

High-frequency ventilation

Liquid ventilation

Use of nitric oxide

Pre-support cardiopulmonary resuscitation

Duration of cardiopulmonary resuscitation

Residual lesions

Palliative versus corrective operation

\section{Post-support variables}

Hemodynamics at 24 hours of support:

Central venous pressure

Right atrial pressure

Left atrial pressure

Systolic blood pressure

Diastolic blood pressure

Mean arterial blood pressure

Arterial blood gases at 24 hours of support:

Oxygen tension

Carbon dioxide tension

$\mathrm{pH}$

Serum bicarbonate

Mixed venous oxygen saturation

Ventilator settings at 24 hours of support:

Inspired oxygen fraction

Peak inspiratory pressure

Positive end-expiratory pressure

Tidal volume

Ventilatory rate

Blood product transfusions during support:

Packed red blood cells

Whole blood

Fresh frozen plasma

Cryoprecipitate

Estimated blood loss on support

Number of re-explorations for bleeding

Use of aminocaproic acid

Inotropic doses at 24 hours of support:

Dopamine 


Amrinone
Dobutamine
Epinephrine
Complication by organ system:
Cardiovascular
Pulmonary
Gastrointestinal
Renal
Infectious
Hemorrhagic
Mechanical
Miscellaneous:
Urine output (over the first 24 hours of support)
Reoperation for residual lesions
Duration chest open
Duration intubated
Carotid reconstruction

\section{Commentary}

Although there is a large experience worldwide with the VAD in adults, and a proliferation of effective support systems, the pediatric experience remains relatively limited. This is due to size and technical considerations, but also to a perception that patients with complex congenital heart disease are unsuitable for univentricular support. Duncan and associates have provided evidence to the contrary, reporting their large pediatric experience with VAD and ECMO for cardiac and cardiopulmonary support. The clinical questions addressed are important for pediatric surgeons. The time frame and the similarities in patient populations and outcome invite commentary and comparison with experience at the Royal Children's Hospital.

Since 1989, we have been using both VAD and ECMO, ${ }^{1-4}$ always employing centrifugal pumps. The centrifugal-pump VAD circuit is straightforward in concept and design, can be rapidly assembled and primed, and requires little technical attention. It is suitable for many cardiac arrest situations. In this format, the centrifugal pump is responsive to changes in the peripheral circulation, allowing fine tuning of inotropic drugs and vasodilators before weaning. The centrifugal pump is probably safer than a roller pump for longterm support outside the operating theater, since it generates constant pressure rather than flow.

The majority of our postoperative support has been with VAD rather than ECMO systems. In practice, at least for intraoperative placement, adequacy of univentricular support can be assessed by converting cardiopulmonary bypass to partial left heart assist, and ceasing gas exchange across the oxygenator. The bypass circuit can then be converted to centrifugalpump VAD or ECMO, as appropriate, on the basis of hemodynamics, oxygenation and carbon dioxide clearance, and acid base status.

We have used the VAD in 53 patients, median age 3.5 months (range 2 days-19 years) and median weight 4 $\mathrm{kg}$ (range 1.9-70 kg). Our results ${ }^{3-5}$ were remarkably similar to those of Duncan and colleagues. The time on VAD was similar for survivors and nonsurvivors $(P=$ .69), with a median of 75 hours. Thirty-eight of 53 children were weaned from VAD $(.72, \mathrm{CL}=.57-.83)$ and 24 of 53 were eventually discharged $(.46, \mathrm{CL}=.31$ $.61)$. Kaplan-Meier survival probability at 1 year was $.44(\mathrm{CL}=.31-.58)$. The post-weaning deaths reflected continued cardiac problems rather than specific VADrelated complications. Age, weight, support time, cardiopulmonary bypass time, ischemic time, univentricular anatomy, and timing of placement (intraoperative versus postoperative) were not strongly associated with survival probability. By comparison, of 40 patients supported with ECMO, 19 were weaned, 3 bridged to transplantation, and 19 eventually discharged from the hospital. Thus, although the weaning probability was better with VAD (.71 vs .48, $P=.014$ ), as in Boston the discharge probability was similar (.46 vs $.48, P=1.0)$. In interpreting these results, one must consider that most of the patients treated with a VAD could have been supported with ECMO, but the reverse would not generally apply.

In the Boston experience, younger patients were more likely to be supported with ECMO than with $\mathrm{VAD}$, because of the complexity of the underlying heart disease and technical considerations. In our own practice, 38 of 53 patients supported with a VAD weighed less than $6 \mathrm{~kg} .{ }^{6}$ The probability of weaning was similar to that for the older patients $(P=.07)$, but the 1 -year survival probability was poorer, primarily related to irreversible cardiac disease. The smallest patient in our series was a 19-day-old, 1.9-kg baby with Taussig-Bing anomaly and arch obstruction, who was placed on VAD support in the postoperative period during a prolonged (1 hour) cardiac arrest and survived with no neurologic sequelae. As would be the case in Boston, we have been obliged to redefine the upper limit for reasonable resuscitation time in children in the era of circulatory support.

Neither VAD nor ECMO in this format is eminently suitable for long-term support. Our longest successful supports have been 120 hours (ECMO) and 144 hours (VAD), although nonsurvivors were well supported metabolically for 384 hours (ECMO) and 428 hours (VAD). Our longest ECMO support for noncardiac indications has been 6 weeks. The usefulness of centrifugal-pump VAD and ECMO as a bridge to trans- 
plantation will depend heavily on the immediate availability of suitable donor hearts, a major problem in many parts of the world. A major limitation is our inability to wean most patients from ventilatory support during centrifugal-pump VAD and ECMO, mobilize them, and make them independent of the intensive care unit.

There are two important questions to be addressed in analyzing this experience. First, do ECMO and VAD as reported herein adequately support the patient when cardiac function is compromised? The answer is clearly yes, although not in every patient, and not indefinitely. Second, does this type of support allow cardiac improvement to occur if improvement would not otherwise have occurred in a given patient? This is a much more complicated question. To ask it another way: What is the effect of patient selection on outcome? As in most areas of cardiac surgery, it is profound. As a group, our VAD patients with anomalous origin of the left coronary artery from the pulmonary artery and those with transposition of the great arteries (especially patients with intact ventricular septum operated on after 3 weeks of age) had a significantly better survival probability than others $(.91, \mathrm{CL}=.59-1.0, P=.04){ }^{7}$ The worst outcome was in patients with technically imperfect repairs, coagulopathy, and those with complex cardiac anomalies that have an expected poor long-term outcome even if VAD and ECMO are not required. Perhaps this type of analysis of indications begs the question. In practice, if a reliable support device were available, almost any child accepted for a cardiac operation would be considered a candidate for support should the need arise.

Centrifugal-pump VAD is not expensive technology. In our own unit, an additional nurse is required when ECMO is used, but not for VAD, during which the child's primary nurse also monitors the VAD circuit (with a perfusionist and cardiac surgeon on call). Consequently, VAD adds only approximately $\$ 250$ per day to normal intensive care unit and hospital expenses, whereas ECMO adds 4 to 5 times that amount.

The availability of paracorporeal pulsatile devices for support of infants and children may render the centrifugal pump system obsolete. Certainly there are several very elegant systems in use in Europe at this time that are proving to be suitable for long-term support, even in very small infants. However, for short-term assistance, the costs involved may place these devices out of the reach of many units, especially those not actively involved in cardiac transplantation.

The results with short-term mechanical support in Boston, Melbourne, and elsewhere strongly support continued application of this strategy for selected children with severe cardiac failure. The simplicity and efficacy of centrifugal-pump VAD should place it in the armamentarium of all units prepared to undertake cardiac operations in children. In the current era, decrement in surgical mortality is very difficult to come by, and Duncan and associates have demonstrated a way of achieving this end.

Tom R. Karl, MS, MD Victoria, Australia

\section{REFERENCES}

1. Karl TR, Horton SB, Mee RBB. Left heart assist for ischaemic postoperative ventricular dysfunction in an infant with anomalous left coronary artery. J Card Surg 1989;4:352-4.

2. Karl TR, Horton SB, Sano S, Mee RBB. Centrifugal pump left heart assist in pediatric cardiac surgery: indications, technique, and results. J Thorac Cardiovasc Surg 1991;102:624-30.

3. Karl TR, Pennington GD. Extracorporeal circulatory support in infants and children. Sem Thorac Cardiovasc Surg 1994;6:15460.

4. Cochrane AD, Horton A, Butt W, Skillington P, Karl TR, Mee RBB. Neonatal and paediatric extracorporeal membrane oxygenation. Aust Assoc J Card Thorac Surg 1992;1:17-22.

5. Karl TR. Circulatory support in children. In: Hetzer R, Hennig E. Loebe M, editors. Mechanical circulatory support. Berlin: Springer; 1997. p. 7-20.

6. Thuys CA, Mullaly RJ, Horton SB, O'Connor EB, Cochrane AD, Brizard CP, et al. Centrifugal ventricular assist in children under 6 kg. Eur J Cardiothorac Surg 1998;13:130-4.

7. Cochrane AD, Coleman DM, Davis AD, Brizard CPR, Wolfe R, Karl TR. Excellent long-term functional outcome after an operation for anomalous left coronary artery from the pulmonary artery. J Thorac Cardiovasc Surg 1998: In press.

$12 / 1 / 95921$ 